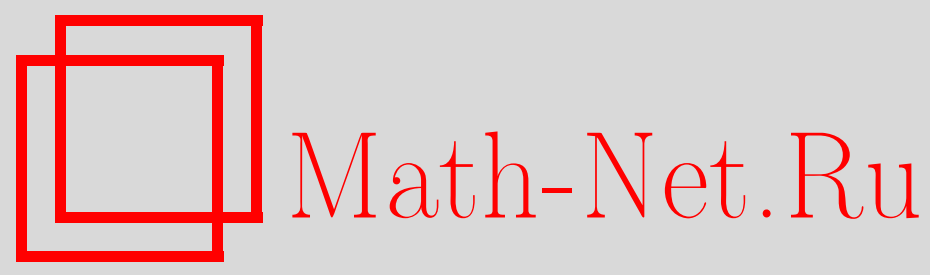

К. О. Бесов, О граничном поведении компонент полигармонических функций, Матем. заметки, 1998, том 64, выпуск 4, 518-530

DOI: https://doi.org/10.4213/mzm1426

Использование Общероссийского математического портала Math-Net.Ru подразумевает, что вы прочитали и согласны с пользовательским соглашением http://www.mathnet.ru/rus/agreement

Параметры загрузки:

IP : 3.82 .47 .9

26 апреля 2023 г., 11:07:17

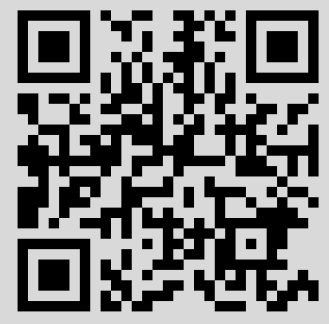




\section{О ГРАНИЧНОМ ПОВЕДЕНИИ КОМПОНЕНТ ПОЛИГАРМОНИЧЕСКИХ ФУНКЦИЙ}

\section{К. О. Бесов}

В работе рассматривается следующее представление полигармонических функций в единичном шаре $D^{m}$ :

$$
f=\Phi_{0}+\left(1-r^{2}\right) \Phi_{1}+\cdots+\left(1-r^{2}\right)^{n-1} \Phi_{n-1}
$$

где $\Phi_{j}$ гармоничны в $D^{m}$. Изучается связь равномерных граничных свойств функции $f$ (гладкость и рост при подходе к границе) с такими же свойствами слагаемых в данном ее представлении. Доказанные теоремы обобщают результаты, полученные Е. П. Долженко в теории полианалитических функций.

Библиография: 6 названий.

Введение. Напомним, что функция $F$, заданная в области $G \subset \mathbb{R}^{m}$ и имеющая непрерьвные частные производные до порядка $2 n$ включительно, назьвается полигармонической порядка $n$ (или $n$-гармонической), если она удовлетворяет уравнению $\Delta^{n} F=0$. Класс всех таких функций обозначим через $H_{n}(G)$. Для единичного шара $D=D^{m}:=\left\{x \in \mathbb{R}^{m}|| x \mid<1\right\}$ всякую функцию $F \in H_{n}:=H_{n}(D)$ можно единственным образом представить в виде

$$
F=\Psi_{0}+r^{2} \Psi_{1}+\cdots+\left(r^{2}\right)^{n-1} \Psi_{n-1}
$$

или, после простых преобразований,

$$
F=f_{0}+f_{1}+\cdots+f_{n-1}, \quad f_{k}=\left(1-r^{2}\right)^{k} \Phi_{k}, \quad 0 \leqslant k \leqslant n-1,
$$

где $r^{2}=x_{1}^{2}+\cdots+x_{m}^{2}$, а $\Phi_{k}, \Psi_{k} \in H:=H_{1}$ - гармонические функции (для удобства мы докажем это в п. 1 , см. также [1]). Очевидно, $\Phi_{n-1}=(-1)^{n-1} \Psi_{n-1}$. Далее слагаемые $f_{k}$ в представлении (2) будем называть компонентами функции $F$, а $\Phi_{k}-$ eе гармоническими компонентами.

Известно, что "хорошие" свойства полигармонической функции не переносятся автоматически на ее гармонические компоненты $\Phi_{k}, \Psi_{k}$. Так, сушествуют бигармонические в $D \subset \mathbb{R}^{2}$ и непрерьвные на $\bar{D}$ функции, гармонические компоненты которых неограничены и почти в каждой точке на $\partial D$ не имеют угловых пределов (см., например, [2]). Ниже изучается связь между равномерными граничными свойствами полигармонической функции $F$ и такими же свойствами слагаемы $x$ в еепредставлении (2). Предварительно напомним некоторые определения.

Пусть $p$ - действительное число. Обозначим через $\langle p\rangle$ наибольшеецелое число, $c m p o-$ го меньшее $p$. Следуюшие классы функций были введены Е. П. Долженко в работе [2].

Работа выполнена при финансовой поддержке Российского фонда фундаментальных исследований, грант № 96-01-01366. 
ОПРЕДЕЛЕНИЕ 1. Ограниченными (соответственно равномерно непрерывными) порядка 0 в области $G$ назовем функции, ограниченные (соответственно равномерно непрерьвные) в этой области. При $p>0$ назовем функцию $f$ ограниченной поряд$\kappa a p$ в области $G$, если у нее в $G$ существуют все частные производные до порядка $\langle p\rangle$ включительно, причем производные порядка меньше $\langle p\rangle$ удовлетворяют условию Lip 1 (считаем $\left.\partial^{0} f / \partial x^{0}:=f\right)$, а все производные порядка $\langle p\rangle-$ условию $\operatorname{Lip}(p-\langle p\rangle)$. Если при этом существуют все частные производные порядка $[p]$ и их модули непрерывности в $G$ суть величины порядка $o\left(t^{p-[p]}\right)(t \rightarrow 0)$, то назовем $f$ равномерно непрерывной порядка $p$ в области $G$. При $p<0$ назовем локально ограниченную в $G$ функцию $f$ ограниченной порядка $p$, если $|f(x)|=O\left(\rho^{p}\right)$ при $\rho=\operatorname{dist}(x, \partial G) \rightarrow 0$; если $f$ непрерьвна в $G,|f(x)|=o\left(\rho^{p}\right)$ при $\rho \rightarrow 0$, то назовем $f$ равномерно непрерывной порядка $p$ в области $G$.

Очевидно, что для гладкой функции $f$ в $D$ условие ограниченности (равномерной непрерьвности) целого порядка $p>0$ эквивалентно ограниченности (равномерной непрерьвности) в $D$ всех ее частных производных порядка $p$. Класс всех функций, имеющих в области $G$ ограниченность порядка $p$, будем обозначать через $M^{p}(G)$, а имеющих равномерную непрерывность порядка $p,-$ через $C U^{p}(G)$. Обобщением классов $M^{p}(G)$ являются классы $M\left(t^{p} \omega(t), G\right)$.

ОПРЕДЕЛЕНИЕ 2. Пусть $p \in \mathbb{Z}$ - целое число, $\omega(t)$ - модуль непрерывности, функция $f$ определена и локально ограничена в области $G$. Скажем, что $f$ обладает ограниченностью уточненного порядка $t^{p} \omega(t)$, и будем писать $f \in M\left(t^{p} \omega(t), G\right)$, если при $p \leqslant-1|f(x)|=O\left(\rho^{p} \omega(\rho)\right)(\rho=\operatorname{dist}(x, \partial G) \rightarrow 0)$ или при $p \geqslant 0 \quad f$ имеет в $G$ все частные производные до порядка $p$ включительно, причем модули непрерьвности всех производных порядка $p$ в $G$ суть $O(\omega(t))(t \rightarrow 0)$, а производные меньших порядков (включая $f$ при $p>0$ ) удовлетворяют условию Lip 1 в $G$.

Под модулем непрерывности мы понимаем функцию $\omega(t)$ со свойствами:

$$
0=\omega(0) \leqslant \omega(u) \leqslant \omega(u+v) \leqslant \omega(u)+\omega(v), \quad u, v \geqslant 0 .
$$

Заметим, что для произвольного модуля непрерывности $\omega$ найдется непрерывньй и выпуклый вверх на $(0,+\infty)$ модуль непрерьвности $\widetilde{\omega}$ такой, что $\frac{1}{3} \widetilde{\omega}(t) \leqslant \omega(t) \leqslant 3 \widetilde{\omega}(t)$ (см., например, [3], где доказан более сильньй результат с условием дважды гладкости $\widetilde{\omega})$. Поэтому далее мы считаем $\omega$ непрерьвным и выпукльм вверх. При этом нас будет интересовать случай, когда $\omega$ подчиняется так назьваемому условию Ульянова-Бари-Стечкина:

$$
\omega(\delta) \geqslant C\left(\int_{0}^{\delta} \frac{\omega(t)}{t} d t+\delta \int_{\delta}^{1} \frac{\omega(t)}{t^{2}} d t\right) \quad \text { для всех } 0<\delta<1 \text {. }
$$

Здесь $C>0$ - некоторая константа, зависящая только от $\omega$.

Из представления (1) в двумерном случае сразу вытекает, что любая полигармоническая функция $F$ в круге $D$ является вешественной частью некоторой полианалитической функции $f$, т.е. такой что $(\partial / \partial \bar{z})^{n} f=0$. Для любой функции $f$ из класса $A_{n}$ полианалитических в круге $D$ функций верно разложение

$$
f(z)=\psi_{0}(z)+\bar{z} \psi_{1}(z)+\cdots+\bar{z}^{n-1} \psi_{n-1}(z)
$$


которое легко преобразуется к виду

$$
\begin{gathered}
f(z)=P(z, \bar{z})+\varphi_{0}(z)+\left(1-|z|^{2}\right) \varphi_{1}(z)+\cdots+\left(1-|z|^{2}\right)^{n-1} \varphi_{n-1}(z), \\
P(z, \bar{z})=\bar{z} P_{1}(z)+\cdots+\bar{z}^{n-1} P_{n-1}(z)
\end{gathered}
$$

где $P_{k}$ - полином от $z$ степени не выше $k-1$, а $\varphi_{k}, \psi_{k}$ - аналитические функции. Долженко получил следующий результат для полианалитических функций (сформулируем его только в случае единичного круга, хотя он остается верным и для более широкого класса областей).

ТЕОРемА ДолЖенко [4]. Пусть функиия $f \in A_{n}$ представлена в виде (4) и $f_{k}(z):=\left(1-|z|^{2}\right)^{k} \varphi_{k}(z), k=0, \ldots, n-1$. Тогда при любом $p \in \mathbb{R}$ справедливь әквиваленции:

$$
\begin{aligned}
f \in M^{p}\left(D^{2}\right) & \Longleftrightarrow f_{k} \in M^{p}\left(D^{2}\right) \quad \forall k, 0 \leqslant k \leqslant n-1, \\
& \Longleftrightarrow \varphi_{k} \in M^{p-k}\left(D^{2}\right) \quad \forall k, 0 \leqslant k \leqslant n-1, \\
f \in C U^{p}\left(D^{2}\right) & \Longleftrightarrow f_{k} \in C U^{p}\left(D^{2}\right) \quad \forall k, 0 \leqslant k \leqslant n-1, \\
& \Longleftrightarrow \varphi_{k} \in C U^{p-k}\left(D^{2}\right) \quad \forall k, 0 \leqslant k \leqslant n-1 .
\end{aligned}
$$

Ниже доказывается аналогичная теорема для полигармонических функций.

\section{1. Вспомогательные утверждения.}

ПРЕДЛОЖЕНИЕ 1. Пусть $m$ - натуральное число. Тогда

1) $\Delta\left(r^{n}\right)=n(m+n-2) r^{n-2}, n>0$;

2) $\Delta\left(h_{1} h_{2}\right)=h_{1} \Delta h_{2}+h_{2} \Delta h_{1}+2\left(\operatorname{grad} h_{1}, \operatorname{grad} h_{2}\right) \quad \forall h_{1}, h_{2} \in C^{2}$;

3) $\Delta\left(r^{n} \Phi\right)=2 n r^{(n-m) / 2} \frac{\partial}{\partial r}\left(r^{(m+n-2) / 2} \Phi\right) \quad \forall \Phi \in H\left(D^{m}\right), n>0$;

4) если $\Phi-$ гармоническая функиия в $D^{m}$, то следующие функции также явяяются гармоническими в $D^{m}$ :

$$
r^{-s} \int_{0}^{r} r^{s-1} \Phi d r, \quad s>0, \quad r^{1-s} \frac{\partial}{\partial r}\left(r^{s} \Phi\right), \quad r^{2-s} \Delta\left(r^{s} \Phi\right), \quad s \geqslant 0 .
$$

Здесь и далее, запись $\int_{0}^{r} d r$ означает интегрирование по отрезку, соединяющему начало координат с точкой $x, r^{2}=x_{1}^{2}+\cdots+x_{m}^{2}$, и результат является функцией точки $x$.

ДокАЗАТЕЛЬСТво. Утверждения 1) и 2) проверяются непосредственно; 3) следует из 2). Докажем 4). Поскольку функция $\Phi$ гармонична в $D$, ее ряд Тейлора с центром в нуле равномерно сходится к ней на любом меньшем (концентрическом) шаре. Группируя члены соответствующим образом, получим ряд из однородных гармонических полиномов вида

$$
\sum_{n_{1}+\cdots+n_{m}=n} C_{n_{1} \ldots n_{m}} r^{n}\left(\cos \theta_{1}\right)^{n_{1}} \cdots\left(\cos \theta_{m}\right)^{n_{m}},
$$

где $\cos \theta_{l}=x_{l} / r$ - направляющие косинусы вектора $\vec{r}$. На каждый такой полином операторы, выраженные формулами (5), действуют как умножение на не слишком быстро растуший (с ростом $n$ ) положительный коэффициент. Интегрируя либо дифференцируя почленно, получаем новые ряды из гармонических полиномов, сходящиеся во всем шаре $D$ к некоторой гармонической функции $\Psi$. Предложение 1 доказано. 
Лемма 1 [5]. Для полигармонической функиии $F \in H_{n}\left(D^{m}\right), m \in \mathbb{N}$, справедливо представление (2):

$$
F=\Phi_{0}+\left(1-r^{2}\right) \Phi_{1}+\cdots+\left(1-r^{2}\right)^{n-1} \Phi_{n-1},
$$

где $\Phi_{k} \in H$ - гармонические функиии.

ДокАЗАТЕЛЬСтво. Достаточно показать, что можно найти гармоническую функцию $\Phi$ такую, что $F-\left(1-r^{2}\right)^{n-1} \Phi \in H_{n-1}$, т.е. $\Delta^{n-1}\left(\left(1-r^{2}\right)^{n-1} \Phi\right)=\Delta^{n-1} F$, что равносильно равенству $(-1)^{n-1} \Delta^{n-1}\left(r^{2 n-2} \Phi\right)=\Delta^{n-1} F$, или в силу предложения 1

$$
\sum_{j=0}^{n-1} C_{j} r^{j} \frac{\partial^{j} \Phi}{\partial r^{j}}=\Delta^{n-1} F \in H(D) .
$$

Теперь легко найти функцию $\Phi$ в виде суммы ряда однородных гармонических полиномов. Действительно, для однородного полинома $P$ операция

$$
r^{j} \frac{\partial^{j} P}{\partial r^{j}}
$$

есть умножение на положительную константу. Лемма 1 доказана.

ЗАМЕЧАНИЕ. Из леммы 1 видно, что любую полигармоническую в единичном шаре функцию $F$ можно разложить в степенной ряд, равномерно сходящийся на всяком компакте $K \subset D$.

Лемма 2. Пусть функиия $f \in H^{n}$ представлена в виде (2). Тогда ее последняя гармоническая компонента имеет вид

$$
\Phi_{n-1}=(-1)^{n-1} \frac{r^{2-n-m / 2}}{4^{n-1}(n-1) !} \underbrace{\int_{0}^{r} d r \cdots \int_{0}^{r}}_{n-1} r^{m / 2-1} \Delta^{n-1} f d r .
$$

ЗАмЕЧАнИЕ. С помошью формулы Коши для повторных интегралов равенство (6) можно переписать в виде

$$
\Phi_{n-1}=(-1)^{n-1} \frac{r^{2-n-m / 2}}{4^{n-1}(n-1) !(n-2) !} \int_{0}^{r}(r-\rho)^{n-2} \rho^{m / 2-1} \Delta^{n-1} f d \rho .
$$

ДокАЗАТЕльСтво. С учетом утверждений 3) и 4) предложения 1 имеем

$$
\begin{aligned}
\Psi(x): & =(-1)^{n-1} \Delta^{n-1} f(x)=\Delta^{n-1}\left(\left(r^{2}-1\right)^{n-1} \Phi_{n-1}(x)\right) \\
& =\Delta^{n-1}\left(r^{2 n-2} \Phi_{n-1}(x)\right)=\Delta\left(r^{2} \frac{\Delta^{n-2}\left(r^{2 n-2} \Phi_{n-1}(x)\right)}{r^{2}}\right) \\
& =4 r^{1-m / 2} \frac{\partial}{\partial r}\left(r^{m / 2} \frac{\Delta^{n-2}\left(r^{2 n-2} \Phi_{n-1}(x)\right)}{r^{2}}\right) \\
& =4 r^{1-m / 2} \frac{\partial}{\partial r}\left(r^{m / 2-2} \Delta^{n-2}\left(r^{2 n-2} \Phi_{n-1}(x)\right)\right) .
\end{aligned}
$$


Отсюда вытекает, что

$$
\Delta^{n-2}\left(r^{2 n-2} \Phi_{n-1}\right)=\frac{r^{2-m / 2}}{4} \int_{0}^{r} r^{m / 2-1} \Psi(x) d r .
$$

С другой стороны,

$$
\begin{aligned}
\Delta^{n-2}\left(r^{2 n-2} \Phi_{n-1}\right) & =\Delta\left(r^{4} \frac{\Delta^{n-3}\left(r^{2 n-2} \Phi_{n-1}\right)}{r^{4}}\right) \\
& =8 r^{2-m / 2} \frac{\partial}{\partial r}\left(r^{m / 2+1} \frac{\Delta^{n-3}\left(r^{2 n-2} \Phi_{n-1}\right)}{r^{4}}\right)
\end{aligned}
$$

Учитьвая (7), получим

$$
\Delta^{n-3}\left(r^{2 n-2} \Phi_{n-1}\right)=\frac{r^{3-m / 2}}{4 \cdot 8} \int_{0}^{r} d r \int_{0}^{r} r^{m / 2-1} \Psi d r
$$

Продолжая процесс, придем к (6). Лемма 2 доказана.

Существенную роль в наших рассуждениях будет играть еще одна характеристика функции, предложенная Долженко, - ореольный модуль непрерьвности порядка $q$.

ОПРЕДЕЛЕниЕ 3. Для числа $R>0$ и функции $f$, заданной в окрестности точки $x \in \mathbb{R}^{m}$ и интегрируемой по Лебегу на сфере $S=S(x, R)=\left\{y \in \mathbb{R}^{m}|| y-x \mid=R\right\}$, назовем

$$
\Delta_{R} f(x):=\frac{1}{\mu S} \int_{S}(f(y)-f(x)) d \mu(y)
$$

ореольной разностью радиуса $R$ функиии $f$ в точке $x$ ( $\mu$-обьчная мера Лебега на сфере $S, \mu S$ - плошадь сферы).

Известно, что если функция $f$ дважды гладка вблизи точки $x$, то

$$
\lim _{R \rightarrow 0} R^{-2} \Delta_{R} f(x)=\frac{1}{2 m}\left(\frac{\partial^{2} f(x)}{\partial x_{1}^{2}}+\cdots+\frac{\partial^{2} f(x)}{\partial x_{m}^{2}}\right)=\frac{1}{2 m} \Delta f(x) .
$$

Если для функции $f$ в точке $x$ определена $n$-я степень оператора $\Delta_{R}$, выражение $\Delta_{R}^{n} f(x)$ будем называть $n$-й ореольной разностью функиии $f$ радиуса $R$ в точке $x$.

Назовем ореольным модулем непрерывности порядка $q, q \in \mathbb{N}$, функции $f$, заданной в области $G \in \mathbb{R}^{m}$, функцию

$$
\omega_{\mathrm{or}}^{(q)}(f, G, \delta):=\sup \left\{\left|\Delta_{R}^{q} f(x)\right|\right\}, \quad 0<\delta<\infty, \quad \omega_{\mathrm{or}}^{(q)}(f, G, 0)=0,
$$

где супремум берется по всем парам $(x, R)$ таким, что $0<R \leqslant \delta$, при этом замкнутьй круг радиуса $q R$ с центром в $x$ лежит в $G$. Легко доказьвается следующее

ПрЕДЛОЖЕНИЕ 2 (Е.П. ДолЖенко). Если $f \in M^{p}(G), \operatorname{mo} \omega_{\mathrm{or}}^{(q)}(f, G, \delta)=O\left(\delta^{s}\right)$, $\delta \rightarrow 0$, где $s=\min \{p, 2 q\}$. Ecлu $f \in C U^{p}(G), \operatorname{mo} \omega_{\text {or }}^{(q)}(f, G, \delta)=o\left(\delta^{p}\right)+O\left(\delta^{2 q}\right)$. 
Лемма 3. Пусть $F \in H_{n}\left(D^{m}\right)$. Тогда

$$
\left|\Delta^{n-1} F(x)\right| \leqslant C \rho^{2(1-n)} \omega_{\mathrm{or}}^{(n-1)}\left(F, D^{m}, \frac{\rho}{n}\right), \quad x \in D^{m}, \quad \rho=\operatorname{dist}\left(x, \partial D^{m}\right),
$$

где C - константа, зависящая только от $n$ и $\mathrm{m}$.

ДокАЗАТЕЛЬСтво. Запишем функцию $F$ в виде (1). Тогда

$$
\begin{aligned}
\Delta_{R} F(0) & =\frac{1}{\mu S} \int_{S(0, R)}\left(\sum_{k=0}^{n-1} r^{2 k} \Psi_{k}(y)-F(0)\right) d \mu(y) \\
& =\sum_{k=1}^{n-1} \frac{R^{2 k}}{\mu S} \int_{S} \Psi_{k}(y) d \mu(y)=\sum_{k=1}^{n-1} R^{2 k} \Psi_{k}(0) .
\end{aligned}
$$

Имеем

$$
\Psi_{0}(0)=F(0), \quad \Psi_{1}(0)=\frac{1}{2 m} \Delta F(0), \quad \ldots, \quad \Psi_{n-1}(0)=\frac{1}{\alpha_{n-1}} \Delta^{n-1} F(0),
$$

где

$$
\alpha_{k}=\Delta^{k} r^{2 k}=\frac{(2 k) ! !(m+2 k-2) ! !}{(m-2) ! !}, \quad k \in \mathbb{N}
$$

и

$$
\Delta_{R} F(0)=\sum_{k=1}^{n-1} R^{2 k} \frac{\Delta^{k} F(0)}{\alpha_{k}} .
$$

Поскольку мы можем сдвигом любую точку перевести в нуль, это равенство справедливо во всем круге $D$ :

$$
\Delta_{R} F(x)=\sum_{k=1}^{n-1} R^{2 k} \frac{\Delta^{k} F(x)}{\alpha_{k}}, \quad \alpha_{1}=2 m .
$$

Отсюда видно, что старший член (при $k=1)$ равен $\frac{1}{2 m} R^{2} \Delta F(x)$ и

$$
\Delta_{R}^{n-1} F(x)=\frac{1}{(2 m)^{n-1}} R^{2 n-2} \Delta^{n-1} F(x),
$$

откуда немедленно вытекает (8). Лемма 3 доказана.

Лемма 4 (Е.П. Долженко). Пусть $D:=D^{m} \subset \mathbb{R}^{m}, m \in \mathbb{N}, j, p$ - иелье иисла, $j>p, j \geqslant 1, \omega-$ модуль непрерьвности, подчиняющийся условию (3), функиия $f$ имеет в $D$ непрерьвные частные производные всех порядков не больше $j$. Тогда

1) если производные порядка $j$ принадлежат классу $M\left(t^{p-j} \omega(t), D\right)$, то $f$ лежит в $M\left(t^{p} \omega(t), D\right)$;

2) если производные порядка $j$ принадлежсат классу $M^{p-j}(D)\left(\right.$ или $\left.C U^{p-j}(D)\right)$, где $p$ - число отрицательное или положительное нечелое, то $f$ лежит в $M^{p}(D)\left(\right.$ соответственно в $\left.C U^{p}(D)\right)$.

Следуюшие леммы распространяют известные результаты из теории полианалитических функций на полигармонические функции. 
ЛЕмма 5. Пусть функиия $\Phi$ гармонична в $D^{m}, p$ - действительное число, $k$ - натуральное. Тогда для принадлежности $(k+1)$-гармонической функции $g(x)=\left(1-r^{2}\right)^{k} \Phi(x) \kappa M^{k+p}\left(D^{m}\right)$ или $C U^{k+p}\left(D^{m}\right)$ необходима и достаточна принадлехсность $\Phi \kappa M^{p}\left(D^{m}\right)$ или $C U^{p}\left(D^{m}\right)$ соответственно.

Лемма 6. Пусть $f \in H_{n}\left(D^{m}\right) \cap M^{p}\left(D^{m}\right)$. Тогда при кажсдом $j \in \mathbb{N}$ все производные порядка $ј$ функиии $f$ принадлежсат $M^{p-j}\left(D^{m}\right)$. Ecли $f \in H_{n}\left(D^{m}\right) \cap C U^{p}\left(D^{m}\right)$, то производные порядка $ј$ принадлежсат $C U^{p-j}\left(D^{m}\right)$.

ЗАмЕчАНИЕ. Из леммы 6 и достаточной части леммы 5 вытекает сформулированная во введении теорема Долженко. Действительно, если функция $f \in A_{n} \cap M^{p}\left(D^{2}\right)$ представлена в виде (4), то $f \in H_{n}$ и

$$
z^{n-1} \varphi_{n-1}(z)=(-1)^{n-1}(n-1) !\left(\frac{\partial}{\partial \bar{z}}\right)^{n-1} f(z) \in M^{p-n+1} .
$$

Отсюда $\varphi_{n-1} \in M^{p-n+1}$, и по лемме $5\left(1-|z|^{2}\right)^{n-1} \varphi_{n-1}(z) \in M^{p}$. Это же рассуждение проходит и для классов $C U^{p}$.

ДокАЗАТЕЛЬСТВО ЛЕМм 5 и 6 проведем только для классов $M^{p}$. Все рассуждения без существенных изменений переносятся на случай $C U^{p}$. Пусть $\mu-(m-1)$-мерная лебегова мера на сфере $S=S\left(x^{0}, R\right)$. Тогда для функции $\Phi$, гармонической на замкнутом шаре $D\left(x^{0}, R\right)$ с центром $x^{0}$ и радиусом $R$, по формуле Пуассона (см. [6, гл. IV, § 2]) имеем

$$
\begin{aligned}
\frac{\partial \Phi}{\partial x_{l}}\left(x^{0}\right) & =\left.\left(\frac{\partial}{\partial x_{l}}\right) \frac{R^{m-2}}{\mu S} \int_{S} \frac{R^{2}-\left|x-x^{0}\right|^{2}}{\left(|x-y|^{2}\right)^{m / 2}}\left(\Phi(y)-\Phi\left(x^{0}\right)\right) d \mu(y)\right|_{x=x^{0}} \\
& =-\frac{R^{m-2}}{\mu S} \int_{S} \frac{\frac{m}{2} R^{2} 2\left(x^{0}-y\right)_{l}}{\left(\left|x^{0}-y\right|^{2}\right)^{m / 2+1}}\left(\Phi(y)-\Phi\left(x^{0}\right)\right) d \mu(y) \\
& =-\frac{R^{m-2}}{\mu S} \int_{S} \frac{m R^{2}\left(x^{0}-y\right)_{l}}{R^{m+2}}\left(\Phi(y)-\Phi\left(x^{0}\right)\right) d \mu(y) \\
& =\frac{m}{R^{2} \mu S} \int_{S}\left(x^{0}-y\right)_{l}\left(\Phi(y)-\Phi\left(x^{0}\right)\right) d \mu(y) .
\end{aligned}
$$

Отсюда

$$
\left|\frac{\partial \Phi}{\partial x_{l}}(x)\right| \leqslant \frac{m}{R} \sup _{|y-x|=R}\{|\Phi(x)-\Phi(y)|\} .
$$

С помощю неравенства (9) легко получить, что производные первого порядка гармонической функции $\Phi \in M^{p}(D)$ лежат в $M^{p-1}$. Действительно, при $p<1$ положим в $(9)$ $R=\frac{1}{2} \operatorname{dist}(x, \partial D)$, а при $p \geqslant 1$ это следует из определения классов $M^{p}$.

Теперь установим достаточность условий леммы 5 . Если $p+k \leqslant 0$, то $\Phi \in M^{p}(D) \Longrightarrow$ $g \in M^{p+k}(D)$ по определению. Пусть $p+k>0$. Обозначим через $q$ наименьшее целое число $\geqslant p+k$. Пользуясь только что установленным свойством производных гармонической функции, для любого мультииндекса $\alpha,|\alpha|=q$, получим

$$
\frac{\partial^{|\alpha|} g}{\partial x^{\alpha}}(x)=\sum_{0 \leqslant \beta \leqslant \alpha} C_{\beta} \frac{\partial^{|\beta|}}{\partial x^{\beta}}\left(\left(1-r^{2}\right)^{k}\right) \frac{\partial^{|\alpha-\beta|} \Phi}{\partial x^{\alpha-\beta}}(x) \in M^{p+k-q}(D) .
$$

Отсюда $g \in M^{p+k}(D)$ (при целом $p$ по определению, а при остальных по лемме 4). 
Доказательство леммы 6 проведем для случая $j=1$ и $p<1$. Случай $p \geqslant 1$ при $j=1$ тривиален, а утверждение леммы для производных высших порядков легко получить итерацией. Итак, пусть $f \in H_{n}(D) \cap M^{p}(D)$ представлена в виде (2). Согласно лемме 3 и предложению $2 \Delta^{n-1} f \in M^{p-2 n+2}$. Каждое интегрирование в (6) улучшает класс на единицу (т.е. если функция была из $M^{s-1}$, то после интегрирования она станет класса $M^{s}$ при условии $s<0$ ). Поскольку множители вида $r^{\lambda}$ не влияют на принадлежность классу $M^{s}$, получаем $\Phi_{n-1} \in M^{p-n+1}$, что вместе с достаточным условием леммы 5 дает $\left(1-r^{2}\right)^{n-1} \Phi_{n-1} \in M^{p}$. Поэтому и $(n-1)$-гармоническая функция $f-\left(1-r^{2}\right)^{n-1} \Phi_{n-1}$ принадлежит $M^{p}$. По-доказанному, функция $\Phi_{n-2}$ лежит в $M^{p-n+2}$, а значит, $\left(1-r^{2}\right)^{n-2} \Phi_{n-2} \in M^{p}$. Продолжая этот процесс, получим $\Phi_{0} \in M^{p}$. Итак,

$$
f \in H_{n} \cap M^{p}(D), \quad p<1, \Longleftrightarrow \Phi_{k} \in M^{p-k}, \quad 0 \leqslant k \leqslant n-1 .
$$

Пользуясь утверждением леммы 6 для гармонических функций (уже доказанным), имеем

$$
\Phi_{k} \in M^{p-k} \Longrightarrow \frac{\partial}{\partial x_{l}}\left(\left(1-r^{2}\right)^{k} \Phi_{k}\right) \in M^{p-1}, \quad 0 \leqslant k \leqslant n-1, \Longrightarrow \frac{\partial f}{\partial x_{l}} \in M^{p-1},
$$

что и требовалось. Лемма 6 доказана.

Наконец, докажем необходимость условий леммы 5. Напомним обозначения: $g(x)=$ $\left(1-r^{2}\right)^{k} \Phi(x) \in H_{k+1} \cap M^{p+k}(D)$. Докажем, что $\Phi \in M^{p}$, "индукцией" по $p$. По лемме $6 \Delta^{k} g \in M^{p-k}$, и при $p<0$ согласно лемме $2 \Phi \in M^{p}$. Предположим, что при фиксированном $k$ мы доказали лемму 5 для всех $p<p_{0}\left(p_{0} \in \mathbb{N} \cup\{0\}\right)$, докажем ее для всех $p, p_{0} \leqslant p<p_{0}+1$.

Выберем пару декартовых координат $x_{j_{1}}, x_{j_{2}}$ и введем в плоскости $O X_{j_{1}} X_{j_{2}}$ полярные координаты $R$ и $\theta$, оставив неизменньми координаты $x_{l}, l \neq j_{1}, j_{2}$. Тог да по лемме 6 $\partial g / \partial \theta=\left(1-r^{2}\right)^{k}(\partial \Phi / \partial \theta) \in M^{p+k-1}$. По предположению индукции $\left(p-1<p_{0}\right)$

$$
\frac{\partial \Phi}{\partial \theta} \in M^{p-1}
$$

(мы воспользовались тем, что $\partial \Phi / \partial \theta$-гармоническая функция). Придавая индексам $j_{1}$ и $j_{2}$ всевозможные различные значения, убедимся в справедливости утверждения (10) для других "полярных" углов $\theta$. Сопоставим каждому дифференциальному оператору $\partial / \partial \theta$ векторное поле на сфере радиуса $r$ с центром в нуле. Разобьем сферу на несколько карт, в каждой из которых выберем из имеющегося набора векторных полей $m-1$, образуюших базис, и введем локальную систему координат $\theta_{1}, \ldots, \theta_{m-1}$. Очевидно, что векторные поля, соответствуюшие операторам $\partial / \partial \theta_{l}$, разлагаются по этому базису, и утверждение (10) остается в силе для производных $\partial \Phi / \partial \theta_{l}, l=1, \ldots, m-1$.

Пусть $D_{0}:=D \backslash \frac{1}{2} D-$ шар $D$ с вькинутой “маленькой" окрестностью нуля. В силу предложения 2

$$
\begin{gathered}
\left(\frac{\partial}{\partial r}\right)^{\nu}\left(r^{k+m / 2-1} \Phi\right)=\frac{1}{4^{k} k !} \underbrace{\int_{0}^{r} d r \cdots \int_{0}^{r}}_{\begin{array}{c}
k-\nu \\
\nu=0,1, \ldots, k
\end{array}} r^{m / 2-1} \Delta^{k}\left((-1)^{k} g\right) d r \in M^{p-k}\left(D_{0}\right),
\end{gathered}
$$


так как $\Delta^{k}\left((-1)^{k} g\right) \in M^{p-k}$, а интегрирование не может ухудшить класс функции. Это дает

$$
\left(\frac{\partial}{\partial r}\right)^{k} \Phi=\left(\frac{\partial}{\partial r}\right)^{k}\left(r^{1-k-m / 2}\left(r^{k+m / 2-1} \Phi\right)\right) \in M^{p-k}\left(D_{0}\right) .
$$

Если $p<k$, то за счет интегрирования имеем $([p]-$ целая часть $p)$

$$
\left(\frac{\partial}{\partial r}\right)^{\nu} \Phi \in M^{p-\nu}\left(D_{0}\right), \quad \nu=[p]+1,[p]+2, \ldots
$$

По лемме 6

$$
\begin{aligned}
\left(\frac{\partial}{\partial r}\right)^{[p]+k}\left((r-1)^{k} \Phi\right) & =(-1)^{k}\left(\frac{\partial}{\partial r}\right)^{[p]+k}\left(\frac{g}{(1+r)^{k}}\right) \in M^{p-[p]}\left(D_{0}\right), \\
\left(\frac{\partial}{\partial r}\right)^{[p]+k}\left((r-1)^{k} \Phi\right) & =\sum_{j=0}^{k} C_{j}(r-1)^{k-j}\left(\frac{\partial}{\partial r}\right)^{[p]+k-j} \Phi \\
& =\sum_{j=0}^{k} C_{k-j}(r-1)^{j}\left(\frac{\partial}{\partial r}\right)^{[p]+j} \Phi \in M^{p-[p]}\left(D_{0}\right) .
\end{aligned}
$$

Все слагаемые в последней сумме имеют вид

$$
C_{k-j}(r-1)^{j}\left(\frac{\partial}{\partial r}\right)^{[p]+j} \Phi=C_{k-j} r^{-[p]-j}(r+1)^{-j}\left(r^{2}-1\right)^{j}\left(r^{[p]+j}\left(\frac{\partial}{\partial r}\right)^{[p]+j} \Phi\right) .
$$

В силу (11) и достаточного условия леммы 5 все слагаемые, кроме первого, лежат в $M^{p-[p]}\left(D_{0}\right)$. Тогда и первое слагаемое $(\partial / \partial r)^{[p]} \Phi$ лежит в $M^{p-[p]}\left(D_{0}\right)$ (при $p \geqslant k$ по определению). Все производные порядка $[p]$ функции $\Phi$ выражаются через $(\partial / \partial r)^{[p]}$ и производные, в которых участвует $\partial / \partial \theta_{l}$. Из (10) и определения классов $M^{p}$ заключаем, что $\Phi \in M^{p}\left(D_{0}\right)$, а значит, $\Phi \in M^{p}(D)$. Индукция и вместе с ней доказательство леммы 5 завершены.

2. Основные результаты. Как будет показано в этом пункте, обобщение теоремы Долженко с простой заменой класса $A_{n}$ и представления (4) соответственно на класс $H_{n}$ и представление (2) неверно даже для бигармонических функций в $\mathbb{R}^{2}$. Однако, если исходная полигармоническая функция $f$ взята из $M^{p}(D)$, то при $p \notin \mathbb{N}$ ее компоненты также лежат в $M^{p}(D)$, а при $p \in \mathbb{N}$ ее компоненты принадлежат классу $M^{q}(D)$ для всех $q<p$.

Рассмотрим сначала полигармонические функции на плоскости.

ТЕОрема 1. Пусть функиия $f \in H_{n}\left(D^{2}\right)$ представлена в виде (2). Тогда при всех действительных $p \neq 1, \ldots, 2 n-2$ справедливы әквиваленции:

$$
\begin{aligned}
f \in M^{p}(D) & \Longleftrightarrow f_{k} \in M^{p}(D) \quad \forall k, 0 \leqslant k \leqslant n-1, \\
f \in C U^{p}(D) & \Longleftrightarrow f_{k} \in C U^{p}(D) \quad \forall k, 0 \leqslant k \leqslant n-1 .
\end{aligned}
$$

При $p=1, \ldots, 2 n-2$ утверждение (12) неверно. 
ДОкАЗАТЕЛЬСТвО. Пусть

$$
f \in H_{n}\left(D^{2}\right) \cap M^{p}\left(D^{2}\right), \quad f_{k}(z)=\left(1-|z|^{2}\right)^{k} \Phi_{k}(z), \quad k=0, \ldots, n-1 .
$$

Как обычно, $z=x+i y \in \mathbb{R}^{2}$. Пусть $p \neq 1, \ldots, 2 n-2$. Преобразуем равенство (6) к виду

$$
\Phi_{n-1}(z)=2 \operatorname{Re}\left(\frac{(-4)^{1-n}}{(n-1) !} \frac{1}{z^{n-1}} \int_{0}^{z} d z_{1} \cdots \int_{0}^{z_{n-1}}\left(\frac{\partial}{\partial z_{n}}\right) \Delta^{n-1} f\left(z_{n}\right) d z_{n}\right)+C .
$$

Справедливость этого равенства легко установить для однородных полигармонических полиномов, следовательно, оно верно для всех $f \in H_{n}\left(D^{2}\right)$. Отметим, что под интегралом стоит аналитическая функция, т.е. результат не зависит от путей интегрирования. По лемме 6 подынтегральное выражение лежит в $M^{p-2 n+1}$. Оператор $\int_{0}^{z}$ переводит аналитическую функцию класса $M^{s}\left(D^{2}\right)$ в функцию класса $M^{s+1}\left(D^{2}\right)$ для всех $s \neq-1$ (см. лемму 4), т.е. получаем $\Phi_{n-1} \in M^{p-n+1}$ и $f_{n-1} \in M^{p}$ по лемме 5. Поэтому и $(n-1)$-гармоническая функция $f-f_{n-1} \in M^{p}$. Продолжая процесс далее, увидим, что все компоненты функции $f$ лежат в $M^{p}\left(D^{2}\right)$.

Аналогичными рассуждениями можно показать справедливость первой части теоремы для классов $C U^{p}$.

Построим функцию $f \in H_{2}\left(D^{2}\right) \cap C U^{1}\left(D^{2}\right) \subset M^{1}\left(D^{2}\right)$, компоненты которой не лежат ни в $M^{1}$, ни в $C U^{1}$. Положим $\Psi(z)=\arg \ln (z-1)=\operatorname{Im} \ln \ln (z-1), \Psi(1)=\arg (-1)$ (у логарифма можно взять произвольную ветвь, определенную в плоскости с разрезом вдоль положительной части вещественной оси).

Легко проверить, что гармоническая функция $\Psi$ (равномерно) непрерывна на $\bar{D}^{2}$, а сопряженная к ней даже неограничена там. Пусть

$$
f(z)=y \arg \ln (z-1), \quad f \in C U^{1} \cap H_{2} .
$$

Тогда

$$
\begin{aligned}
f(z) & =\operatorname{Im}\left(\frac{z-\bar{z}}{2 i} \ln \ln (z-1)\right) \\
& =-\frac{1}{2} \operatorname{Re}\left(z \ln \ln (z-1)-\bar{z} \ln \ln (-1)-|z|^{2} \frac{\ln \ln (z-1)-\ln \ln (-1)}{z}\right), \\
\Phi_{1}(z) & =\frac{1}{2} \operatorname{Re} \frac{\ln \ln (z-1)-\ln \ln (-1)}{z}=\frac{1}{2|z|^{2}} \operatorname{Re}(\bar{z}(\ln \ln (z-1)-\ln \ln (-1))) \\
& =\frac{x\left(\ln |\ln (z-1)|-c_{1}\right)-y\left(\arg \ln (z-1)-c_{2}\right)}{2|z|^{2}}
\end{aligned}
$$

$\Phi_{1}$ неограничена и, значит, старшая компонента $\left(1-|z|^{2}\right) \Phi_{1}(z)$ не лежит в $C U^{1}$ (и даже в $M^{1}$ ), в то время как $f \in C U^{1}$.

Для завершения доказательства покажем, что $n$-гармоническая функция

$$
f(z)=\left(1-|z|^{2}\right)^{p-2[p / 2]}\left(|1-z|^{2}\right)^{[p / 2]} \arg \ln (z-1)
$$

лежит в $C U^{p}(D)$ при $p=2, \ldots, 2 n-2$, однако ее компоненты не лежат даже в $M^{p}(D)$. Первьй множитель без изменений переносится на компоненты функции $f$ и не играет существенной роли. При нечетных $p$ он улучшает гладкость на единицу, а при четных 
равен 1. Поэтому достаточно рассмотреть функцию $g(z)=|z-1|^{2 l-2} \arg \ln (z-1)$, $g \in C U^{2 l-2} \cap H_{l}$, и показать, что ее компоненты не лежат в $M^{2 l-2}$. Имеем

$$
g(z)=\operatorname{Im}\left(|z-1|^{2 l-2} \ln \ln (z-1)\right)=\operatorname{Im}\left(\bar{z}^{l-1}(z-1)^{l-1} \ln \ln (z-1)+h(z)\right),
$$

где $h \in A_{l-1}$ не влияет на гармоническую компоненту $\Phi_{l-1}$. Далее,

$$
\begin{aligned}
\Phi_{l-1}(z) & =\operatorname{Im} \frac{(z-1)^{l-1} \ln \ln (z-1)-P_{l-1}(z)}{z^{l-1}} \\
& =\frac{1}{|z|^{2 l-2}} \operatorname{Im}\left(\bar{z}^{l-1}\left((z-1)^{l-1} \ln \ln (z-1)-P_{l-1}(z)\right)\right),
\end{aligned}
$$

где $P_{l-1}$ - начальный отрезок ряда Тейлора функции $(z-1)^{l-1} \ln \ln (z-1)$ с центром в нуле. Легко проверить, что компонента $\left(1-|z|^{2}\right)^{l-1} \Phi_{l-1}(z)$ имеет неограниченные производные порядка $2 l-2$, т.е. она не лежит ни в $C U^{2 l-2}$, ни даже в $M^{2 l-2}$. Теорема 1 доказана.

ТЕОрема 2. Пусть функиия $f \in H_{n}\left(D^{m}\right), m \geqslant 3$, представлена в виде (2). Тогда при всех $p \in \mathbb{R} \backslash \mathbb{N}$ справедливы әквивалениии:

$$
\begin{aligned}
f \in M^{p}(D) & \Longleftrightarrow f_{k} \in M^{p}(D) \quad \forall k, 0 \leqslant k \leqslant n-1, \\
f \in C U^{p}(D) & \Longleftrightarrow f_{k} \in C U^{p}(D) \quad \forall k, 0 \leqslant k \leqslant n-1 .
\end{aligned}
$$

При $p=1, \ldots, 2 n-2$ утверждение (13) неверно.

Нам удалось построить при всех $p \in \mathbb{N}$ примеры бигармонических функций в $D^{m}$, $m \geqslant 3$, опровергаюшие утверждение (13). Однако, это построение слишком объемно и использует совсем другие идеи, так что оно будет изложено в другой работе автора.

ДокАЗАТЕЛЬСТво, как обычно, проведем только для классов $M^{p}$. Если $f \in M^{p}(D)$, то по лемме $6 \Delta^{n-1} f \in M^{p-2 n+2}(D)$. При $p<1$ из (6) следует, что $\Phi_{n-1} \in M^{p-n+1}$, и по лемме $5 f_{n-1}=\left(1-r^{2}\right)^{n-1} \Phi_{n-1} \in M^{p}$. Проводя те же рассуждения для $(n-1)$-гармонической функции $f-f_{n-1}$, получим $f_{n-2} \in M^{p-n+2}, \ldots, f_{0} \in M^{p}$. Таким образом, при $p<1$ теорема доказана. Случай нецелых положительных $p$ будет описан в теореме 3 , в которой мы рассмотрим классы $M\left(t^{p} \omega(t)\right)$ с условием (3) на модуль непрерьвности $\omega$ (в нашем случае $\left.\omega(t)=t^{p-[p]}\right)$.

Далее заметим, что примеры функций, доказывающие вторую часть теоремы 1 , легко переносятся на случай произвольной размерности путем добавления несущественных переменных. Другими словами, для функции, заданной в круге на плоскости, мы строим новую функцию $f$ на шаре в пространстве, значение которой в точке $x$ совпадает со значением исходной функции в точке $\pi(x)$, где $\pi$-проекция на плоскость. Запишем последнюю гармоническую компоненту полигармонической функции $f$ по формуле (6) и рассмотрим сужение этой компоненты на плоскость. Тогда точно так же, как и в двумерном случае, $\int_{0}^{\vec{r}} d s$ в (6) можно преобразовать к виду $\int_{0}^{z} d z$ и убедиться, что последняя компонента не принадлежит к соответствующему классу $M^{p}$.

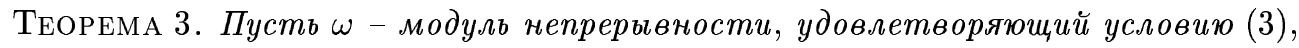
функция $f \in H_{n}\left(D^{m}\right), m \in \mathbb{N}$, представлена в виде (2). Тогда при всех иельх $p$ справедливы әквивалениии:

$$
\begin{aligned}
f \in M\left(t^{p} \omega(t), D\right) & \Longleftrightarrow f_{k} \in M\left(t^{p} \omega(t), D\right) \quad \forall k, 0 \leqslant k \leqslant n-1, \\
& \Longleftrightarrow \Phi_{k} \in M\left(t^{p-k} \omega(t), D\right) \quad \forall k, 0 \leqslant k \leqslant n-1 .
\end{aligned}
$$

Кроме того, при кажсом $j \in \mathbb{N}$ все частные производные порядка $j$ полигармонической функиии $f \in M\left(t^{p} \omega(t), D\right)$ лежат в $M\left(t^{p-j} \omega(t), D\right)$. 
ДокАЗАтЕльСтво. Пусть $f \in M\left(t^{p} \omega(t)\right)$. Покажем, что $\Delta^{n-1} f \in M\left(t^{p-2 n+2} \omega(t)\right)$. Если $p \geqslant 2 n-2$, то это следует из определения классов $M$. Пусть $p<2 n-2$. Тогда по лемме 3 имеем $(\rho:=1-|x|)$

$$
\left|\Delta^{n-1} f(x)\right| \leqslant C \rho^{2-2 n} \omega_{\text {or }}^{n-1}(f, D, \rho) \leqslant C_{1} \rho^{2-2 n} \rho^{p} \omega(\rho) .
$$

Чтобы получить второе неравенство, нужно записать формулу Тейлора для функции $f$ в точке $x$. Оператор $\Delta_{\rho}^{n-1} f$ уничтожит первые $p<2 n-2$ членов. Таким образом, $\Delta^{n-1} f \in M\left(t^{p-2 n+2} \omega(t)\right)$. Из формулы (9) заключаем, что все производные порядка $j$ гармонической функции $\Delta^{n-1} f$ лежат в $M\left(t^{p-2 n+2-j} \omega(t)\right)$.

В силу выпуклости $\omega$ при

$$
u(t):=\int_{0}^{t} \omega(\lambda) d \lambda
$$

имеем

$$
\begin{gathered}
\frac{1}{2} t \omega(t) \leqslant u(t) \leqslant t \omega(t), \\
J:=\int_{\rho}^{1} t^{q-1} \omega(t) d t=u(1)-\rho^{q-1} u(\rho)+(1-q) \int_{\rho}^{1} u(t) t^{q-2} d t, \\
J>0-\rho^{q} \omega(\rho)+\frac{1}{2}(1-q) J, \\
J=\int_{\rho}^{1} t^{q-1} \omega(t) d t<2 \rho^{q} \omega(\rho), \quad q \leqslant-2 .
\end{gathered}
$$

Запишем формулу (6) в виде

$$
\Phi_{n-1}= \pm \frac{r^{2-n-m / 2}}{4^{n-1}(n-1) !} \underbrace{\int_{0}^{r} d r \cdots \int_{0}^{r}}_{n-1} r^{m / 2-1} \Delta^{n-1} f d r .
$$

Пусть $p \geqslant n-3$. Любую производную функции $\Phi_{n-1}$ можно выразить через производные по радиусу $r$ и по углам $\theta_{l}$, причем производная по радиусу "убивает" одно интегрирование в (6), а производная по $\theta_{l}$ вносится под знак интеграла и ухудшает на единицу класс подьнтегральной функции. Оценивая с помощью (14) рост частных производных порядка $p-n+3$ функции $\Phi_{n-1}$, получаем, что все производные порядка $p-n+3$ функции $\Phi_{n-1}$ лежат в $M\left(t^{-2} \omega(t)\right)$, и по лемме $4 \Phi_{n-1} \in M\left(t^{p-n+1} \omega(t)\right)$.

Если $p<n-3$, то аналогичным образом с помощью неравенства (14) можно показать, что $\Phi_{n-1} \in M\left(t^{p-n+1} \omega(t)\right)$.

Оценивая с помощью (9) рост частных производных функции $\left(1-r^{2}\right)^{n-1} \Phi_{n-1}=f_{n-1}$ и применяя лемму 4 , приходим к выводу, что $f_{n-1} \in M\left(t^{p} \omega(t), D\right)$. Проводя те же рассуждения для $(n-1)$-гармонической функции $f-f_{n-1}$, получим первую часть теоремы 3 . Вторая часть непосредственно следует из первой и (9). Теорема 3 доказана.

Теоремы 1 и 2 показывают, что для полигармонических функций в единичном шаре имеется некоторый набор исключительных значений параметра $p$, при которых класс $M^{p}(D)$ не содержит вместе с каждой функцией $f \in H_{n}(D)$ ее компоненты. Ниже мы введем определение несколько иных классов гладкости, которые при $p<0$ совпадают 
c $M^{p}$. Это позволит получить для полигармонических функций теорему без исключительных значений $p$, подобную теореме Долженко. Отметим, что совершенно аналогично классам $\widetilde{M}^{p}$ можно определить классы $\widetilde{C U}{ }^{p}$ и сформулировать для них соответствующую теорему.

Вернемся к теореме Долженко о полианалитических функциях. Отсутствие исключительных значений $p$ связано с тем, что нам не нужно интегрировать какие бы то ни было функции для восстановления по функции $f \in A_{n}$ ее последней аналитической компоненты $\varphi_{n-1}$ :

$$
\varphi_{n-1}(z)=\frac{(-1)^{n-1}(n-1) !}{z^{n-1}}\left(\frac{\partial}{\partial \bar{z}}\right)^{n-1}(f(z)-P(z, \bar{z}))
$$

(см. (4)). В полигармоническом случае одно интегрирование в (6) не всегда улучшает класс гладкости $M^{p}$ на единицу. Именно с учетом исправления этого недостатка мы и вводим новые классы $\widetilde{M}^{p}$.

ОПРЕДЕЛЕНИЕ 4. Будем говорить, что функция $f$, заданная и непрерывная в шаpe $D$, принадлежит классу $\widetilde{M}^{p}$, если при $p<0 f(x)=O\left(\rho^{p}\right), \rho=\operatorname{dist}(x, \partial D) \rightarrow 0$ (т.е. $\left.f \in M^{p}(D)\right)$, а при $p \geqslant 0 f$ имеет в $D$ непрерывные частные производные до порядка $[p]+1$ включительно, причем производные порядка $[p]+1$ растут как $O\left(\rho^{\{p\}-1}\right)$ при $\rho \rightarrow 0$.

ЗАмечАниЕ. По лемме 6 для полигармонических функций имеем $f \in M^{p}(D) \Longrightarrow$ $f \in \widetilde{M}^{p}(D)$, и по лемме 4 при $p \notin \mathbb{N} \cup\{0\} f \in M^{p} \Longleftrightarrow f \in \widetilde{M}^{p}$.

ТЕОрема 4. Пусть функиия $f \in H_{n}\left(D^{m}\right)$ представлена в виде (2). Тогда при любом $p \in \mathbb{R}$ имеют место әквивалениии:

$$
f \in \widetilde{M}^{p} \Longleftrightarrow f_{k} \in \widetilde{M}^{p} \quad \forall k, 0 \leqslant k \leqslant n-1, \Longleftrightarrow \Phi_{k} \in \widetilde{M}^{p-k} \quad \forall k, 0 \leqslant k \leqslant n-1 .
$$

ДокАЗАТЕЛЬСТво непосредственно вытекает из формулы (6) и того, что любая частная производная выражается через производную по радиусу и производные по $\theta_{l}$ $\left(\cos \theta_{l}=x_{l} / r\right)$.

Заметим, что из теоремы 4 не следует ограниченность компонент ограниченной полигармонической функции $f$, которая на самом деле имеет место. Однако этот случай $\left(f \in M^{0}\right)$ содержится в теоремах 1 и 2 .

\section{СПИСОК ЦИТИРОВАННОЙ ЛИТЕРАТУРЫ}

[1] Nicolesco M. Les fonctions polyharmoniques. Paris: Herman, 1936.

[2] Долженко Е. П. О граничном поведении компонент полианалитической функции // Докл. РАH. 1994. Т. 338. № 5. С. 585-588.

[3] Долженко Е.П. Об особых точках непрерывных гармонических функций // Изв. АН СССР. Сер. матем. 1964. Т. 28. №6. С. 1251-1270.

[4] Долженко Е. П. О граничном поведении компонент полианалитической функции // Матем. заметки. 1998. Т. 63. №6. С. 821-834.

[5] Almansi E. Sull'integrazione dell'equazione differenziale $\Delta^{n}=0 / /$ Annali di Matematica. Sèr. 3. 1899. V. 2. P. 1-59.

[6] Курант Р., Гильберт Д. Методы математической физики. Т. 2. М.: Мир, 1964. 